\title{
Hétérogénéité génétique et résistance des vecteurs du paludisme (Anopheles gambiae s.l) aux insecticides en zone cotonnière au Benin
}

\author{
Rock AIKPON ${ }^{1 *}$, Antoine MISSIHOUN ${ }^{1}$, Antoine LOKOSSOU ${ }^{2}$, Gorgias AIKPON ${ }^{2}$, \\ Sahidou SALIFOU ${ }^{2}$, Alexandre DANSI ${ }^{1}$ et Clément AGBANGLA ${ }^{2}$ \\ ${ }^{1}$ Université Nationale des Sciences, Technologies, Ingénierie et Mathématiques, BP 2282 Goho, Benin. \\ ${ }^{2}$ Université d'Abomey-Calavi, BP 526 Cotonou, Benin. \\ *Auteur correspondant ; E-mail : rockypremier@yahoo.fr
}

\section{RESUME}

La résistance des vecteurs du paludisme aux insecticides utilisés dans la lutte anti vectorielle est un obstacle majeur pour les programmes nationaux de lutte contre le paludisme. La présente étude avait pour but d'évaluer la diversité génétique des populations de Anopheles gambiae et la résistance aux insecticides (mutation $K d r$ et Ace-1) en zone cotonnière au Bénin. Deux méthodes de capture ont été utilisées pour la collecte d'échantillon. La capture sur appât humain et la récolte de la faune résiduelle matinale par spray. La PCR SINE a permis de distinguer deux espèces jumelles et d'obtenir les différents génotypes pour les deux gènes. Deux espèces du complexe An. gambiae s.l ont été trouvées en sympatrie (Anopheles coluzzii et Anopheles gambiae). La résistance $k d r$ et $A c e-1$ connaissent un flux saisonnier. L'allèle résistant du gène $k d r$ est fixé dans la population de vecteurs $\left(F_{i s}>0\right)$. Par contre, un déficit de l'allèle résistant est noté au niveau du gène Ace-1 $\left(F_{i s}<0\right)$. (C) 2020 International Formulae Group. All rights reserved.

Mots clés : Hétérogénéité, génétique, résistance, vecteur, paludisme.

\section{Genetic heterogeneity and resistance to insecticides in malaria vector Anopheles gambiae s.l in the cotton growing area in Benin}

\begin{abstract}
Resistance of malaria vectors to insecticides used in vector control is a major threat for national malaria control programs. The purpose of this study was to assess the genetic diversity of Anopheles gambiae populations and resistance to insecticides (Kdr Ace-1 mutation) in the cotton growing area in Benin. Two samples methods were used for mosquito collection: Human Landing catch (HLC) and the residual fauna collection by spray. The SINE PCR allow to identify species the different genotypes resistance genes. Two species of the An. gambiae s.l complex have been found in sympatry (Anopheles coluzzii and Anopheles gambiae). $k d r$ and Ace-1 resistance had seasonal variation trend. The $k d r$ resistance allele is fixed in the vector population $\left(F_{i s}>0\right)$. Moreover, a deficit of the Ace-1 resistance allele was observed $\left(F_{i s}<0\right)$.

(C) 2020 International Formulae Group. All rights reserved.
\end{abstract}

Keywords: Heterogeneity, genetic, resistance, vector, malaria. 


\section{INTRODUCTION}

La lutte acharnée contre le paludisme heurte à l'obstacle de la résistance des parasites aux médicaments antipaludéens (Plowe 2009) et des moustiques vecteurs aux insecticides (Ranson et al., 2011). An. gambiae s.l. constitue le vecteur principal du paludisme en Afrique au sud du Sahara (Taye et al., 2006). Parmi les différentes méthodes couramment utilisées pour contrôler cette maladie, les Moustiquaires Imprégnées d'Insecticides à Longue Durée d'action (MIILD) et la Pulvérisation Intra Domiciliaire (PID) d'insecticides à effet rémanent sont les plus utilisées (Beier et al., 2008; Kelly-Hope et al., 2008). L'utilisation abusive des mêmes classes d'insecticides en agriculture augmente le niveau de résistance (Hemingway et al., 2004). Cette augmentation de la résistance pourrait être liée à la sélection des allèles de résistance qui sont les plus avantageux dans le nouveau milieu créé par cette utilisation abusive de ces insecticides comme mentionné en Afrique de l'Ouest (Corbel et al., 2007; Darriet, 2017 ; Yadouléton et al., 2018).

De récentes études ont montré l'implication de deux gènes ( $K d r$ et Ace-1) dans la résistance des moustiques vecteurs du paludisme à certaines classes d'insecticides comme les carbamates et les pyréthrinoïdes (Bimenya et al., 2010; Aïkpon et al., 2013; Salako et al., 2018). Par ailleurs, des études conduites au Burkina-Faso (Diabate et al., 2002) et au Bénin (Yadouleton et al., 2010) ont montré que chez An. gambiae s.l. la fréquence de l'allèle de résistance $(1014 F)$ du gène $K d r$ est plus élevée dans les zones de culture habituellement soumises à des traitements insecticides qu'en zones rurales où les paysans ne pratiquent que les cultures vivrières ou des produits de consommation locale. Dans le contexte actuel où la résistance aux pyréthrinoïdes et aux cyclodiènes est très largement répandue en Afrique (Ranson et al., 2011; Ahadji-dabla et al., 2014), les études de la diversité des gènes de résistance aux insecticides des populations anophéliennes sont d'une importance majeure pour améliorer les outils de lutte contre An. gambiae, vecteur majeur de plasmodies humaines.

La résistance d'An. gambiae s.l. aux pyréthrinoïdes, seuls insecticides utilisés pour l'imprégnation des moustiquaires est connue au Bénin et bien documentée (Aïkpon et al., 2014 ; Ahadji-dabla et al., 2019) Il apparaît important, au regard de la dynamique qui caractérise les populations d'organismes vivants, de s'intéresser à l'évolution biologique dans le temps et dans l'espace des espèces vectrices comme An. gambiae s.l. afin de mieux gérer la résistance des vecteurs. C'est sur cette base que la présente étude se propose de suivre la dynamique des gènes de résistance $K d r$ et Ace- 1 chez les vecteurs du paludisme (Anopheles gambiae s.l) en zone cotonnière au Bénin. Elle permettra d'évaluer la diversité spécifique et génétique de la population anophélienne et d'évaluer leur niveau de résistance en zone cotonnière

\section{MATERIEL ET METHODES \\ Zone d'étude}

Cette étude a été réalisée de mars à août 2019 dans trois communes du département de l'Atacora (Natitingou, Toukountouna et Boukoumbé) (Figure 1). Le département de l'Atacora est situé au nordouest du Bénin. Il compte neuf communes et couvre une superficie de $31665 \mathrm{~km}^{2}$ caractérisé par un climat tropical dominé par deux saisons : une saison sèche (décembre - mai) et une saison pluvieuse (juillet - novembre) avec une pluviométrie annuelle moyenne comprise entre 1200 et $1350 \mathrm{~mm}$. L'activité économique principale est l'agriculture dominée par la production du coton et du mil dont les rendements sont améliorés par l'usage des engrais chimiques et des pesticides. Dans le cadre de cette étude, deux localités ont été choisies par commune pour l'échantillonnage ; une localité urbaine et une autre périurbaine (Koussantigou et Perma) dans Natitingou, (Tchakalakou et Wabou) dans Toukountouna et (Koutagou et Tassayota) dans Boukoumbé. 


\section{Méthodes expérimentales \\ Echantillonnage des moustiques vecteurs}

Des captures de moustiques adultes ont été faites selon deux méthodes de capture: la capture sur appât humain (Figure 2a) et la récolte de la faune résiduelle par spray matinal dans les habitations humaines (Figure 2b). Le spray matinal consiste à pulvériser à l'aide d'une bombe aérosol les coins ou angles et cachettes des chambres tôt le matin avant l'ouverture des portes et fenêtres afin de recueillir les moustiques quinze minutes de temps après l'épandage sur des draps blancs étalés au sol (Djègbè et al., 2017). La capture sur appât humain, quant à elle, a été faite sur des volontaires munis de lampe torche et de tubes hémolyses. Ils capturent les moustiques se posant sur leurs jambes dénudées avant toute piqûre. Deux équipes de volontaires par commune sont formées grâce à cette technique de capture des moustiques. L'une des équipes est disposée à l'intérieur et l'autre à l'extérieur des ménages choisis.

\section{Caractérisation morphologique du complexe} Anopheles gambiae s.l

L'identification des espèces capturées dans la zone d'étude est la première étape. Elle consiste à l'identification morphologique à la loupe binoculaire à l'aide de clé de détermination établie par Gillies et Coetzee (1987).

\section{Caractérisation moléculaire des populations Anopheles gambiae s.l capturées}

La caractérisation moléculaire passe par les trois étapes suivantes:

Etape 1: Extraction d'ADN génomique du moustique au CTAB 2\%

Les carcasses des moustiques (abdomen, ailes et pattes) ont été broyées dans $200 \mu \mathrm{l}$ de CTAB à $2 \%$. Après $5 \mathrm{mn}$ au bainmarie à $65^{\circ} \mathrm{C}$, le broyat a été mélangé avec 200 $\mu \mathrm{l}$ de chloroforme puis centrifugé à 14000 tours par minute pendant $5 \mathrm{mn}$. Le surnageant a été délicatement récupéré dans un autre tube auquel on ajoute $200 \mu \mathrm{l}$ d'isopropanol puis centrifugé à 12000 tours par minute pendant 15 minutes. La pelote d'ADN obtenue a été conservée avec $200 \mu \mathrm{l}$ d'éthanol à $70 \%$.
L'ensemble a été centrifugé à 14000 tours par minute pendant $5 \mathrm{mn}$.

Le contenu du tube a été délicatement renversé afin de conserver la pelote d'ADN qui était ensuite séché pendant un moment sur la paillasse. Enfin $20 \mu \mathrm{l}$ d'eau bi-distillée ont été ajoutés au pelote d'ADN pour la reconstituer.

\section{Etape 2: Amplification de l'ADN par PCR}

L'identification des espèces se fait par la PCR diagnostic en se servant des amorces $\mathrm{UN}, \mathrm{AG}, \mathrm{AA}$ et AM. L'amorce UN est universelle et s'hybride avec une séquence nucléotidique particulière chez toutes les espèces de moustique. AG forme avec UN le couple d'amorces qui amplifie un fragment nucléotidique précis chez Anopheles gambiae s.l Il en est de même du couple d'amorces UN et AA qui amplifie un fragment nucléotidique particulier chez Anopheles arabiensis. Enfin, le couple d'amorces UN et AM réalise l'amplification nucléotidique d'un fragment uniquement chez Anopheles melas.

Pour différencier Anopheles gambiae s.s. et Anopheles coluzzii la PCR SINE selon le protocole de Santolamazza et al., (2008) est faite avec les couples d'amorces F6 et R6.

Le Tableau 1 présente les séquences nucléotidiques de ces amorces.

Après amplification de l'ADN les tailles attendues sont : An. coluzzii: $479 \mathrm{pb} ; A n$. gambiae s.s: $249 \mathrm{pb} ;$ An. arabiensis: $223 \mathrm{pb}$.

Pour déterminer la mutation $L 1014 \mathrm{~F}$ du gène $K d r$ chez les différentes espèces, la PCR selon le protocole de Martinez-Torres et al., (1998) est faite avec les amorces AgD1, $\mathrm{AgD} 2, \mathrm{AgD} 3$ et AgD4. Le couple d'amorces $\mathrm{AgD} 1 / \mathrm{AgD} 2$ flanque le gène de mutation $K d r$, en amplifiant un produit de 293pb comme contrôle. Le couple d'amorce AgD3/AgD1 s'apparie seulement avec la portion résistante du gène $K d r$ pour amplifier un fragment de 195 pb. Le couple AgD4/AgD2 s'associe uniquement avec la portion du gène sensible en amplifiant un fragment de 137pb. Enfin l'identification de la présence de la mutation G119S se fait grâce aux amorces (Tableau 2) selon le protocole de Weill et al., (2004). 
Etape 3 : Migration de l'amplifia

Les produits d'amplification obtenus ont été migrés sur gel d'agarose $2 \%$. A la fin de la migration, la visualisation de la taille des fragments d'ADN a été faite à l'ultra-violet en comparant les différentes bandes obtenues au marqueur de poids moléculaire 100pb.

\section{Analyses statistiques}

Pour mieux interpréter les résultats, l'ensemble des individus d'une même espèce capturés dans le département de l'Atacora est considéré comme la population d'espèce tandis que celui des individus d'une même espèce capturée par commune est considéré comme sous-population. Plusieurs approches analytiques ont été utilisées pour analyser les données de cette étude. Le logiciel Genepop version 4.2 a été utilisé pour calculer les fréquences alléliques des deux gènes. La $\mathrm{p}$ value associée à chaque fréquence du gène a été calculée avec la fonction de test binomial $\mathrm{du} \operatorname{logiciel} \mathrm{R}$ version 3.3.3. L'équilibre de Hardy-Weinberg a aussi été testé (HWE) pour vérifier s'il y avait panmixie dans les populations d'anophèles des zones d'étude.

Le logiciel Genepop version 4.2 a également permis de calculer certains indices de diversité génétique comme l'hétérozygotie observée $\left(H_{o}\right)$, hétérozygotie attendue $\left(H_{e}\right)$, indice de fixation $\left(F_{I S}\right)$ et la différenciation génétique au sein des populations $\left(F_{S T}\right)$ pour identifier les causes probables d'un éventuel écart par rapport à la panmixie à l'aide de (Weir et Cockerham 1984). L'indice de fixation $\left(F_{I S}\right)$ permet de quantifier la différence par rapport à la panmixie. Si $F i s<0$, la population exprime un excès d'hétérozygotes, mais si $F_{I S}>0$, il présente un déficit en hétérozygotes. Les critères utilisés pour évaluer la différenciation génétique au sein des populations sont ceux définis par Hartl. La différenciation génétique est faible si la valeur $F_{S T}$ est inférieure ou égale à 0,05 , modérée quand sa valeur est comprise entre $[0,05 ; 0,15]$ et importante quand il est compris entre $[0,15 ; 0,25]$. Cette différenciation est très importante si la valeur $F_{S T}$ est supérieure à 0,25 .

Tableau 1 : Amorces utilisées pour identifier les espèces du complexe Anopheles gambiae.

\begin{tabular}{lll}
\hline $\begin{array}{l}\text { Séquence nucléotidique des amorces du PCR } \\
\text { diagnostic }\end{array}$ & $\begin{array}{l}\text { Séquence } \\
\text { diagnostic SINE (Santolamazza et al. 2008) }\end{array}$ \\
\cline { 1 - 1 } UN 5'-GTGTGCCGCTTCCTCGATGT-3' & F6 : TCG CCT TAG ACC TTG CGT TA \\
\hline AG 5'-CTGGTTTGGTCGGCACGTTT-3' & \multirow{2}{*}{ R6 : CGC TTC AAG AAT TCG AGA TAC } \\
\cline { 1 - 2 } AA 5'-AAGTGTCCTTCTCCATCCTA-3' & \\
\hline AM 5'-GTGACCAACCACTCCCTTGA3' & \\
\hline PCR : Polymerase Chain Reaction ; SINE : short INterspersed Elements.
\end{tabular}

Tableau 2 : Séquences nucléotidiques des amorces d'identification des allèles mutés.

\begin{tabular}{lc}
\hline $\begin{array}{l}\text { Les séquences nucléotidiques des } \\
\text { amorces pour la mutation } \mathbf{K} d \boldsymbol{r} \\
\text { (Martinez-Torres } \text { et al. 1998) }\end{array}$ & $\begin{array}{c}\text { Les séquences nucléotidiques des } \\
\text { amorces pour la mutation } \text { Ace-1 } \\
\text { (Weill } \text { et al. 2004). }\end{array}$ \\
\hline Agd1 :5'-ATAGATTCCCGACCATG-3' & Ex3AGdir : 5'-GATCGTGGACACCGTGTTCG-3' \\
\hline Agd2 : 5'-ACAAGGATGATGAACC-3' & Ex3AGrev:5'- AGGAT GGCCCGCTGGAACAG-3' \\
\hline Agd3: 5'-AATTTGCATTACTTACGACA-3' & \\
\hline Agd4:5'-CTGTAGTGATAGGAAATTTA-3' & \\
\hline
\end{tabular}

Kdr : knockdown resistance; Ace : Acetylcholinesterase. 


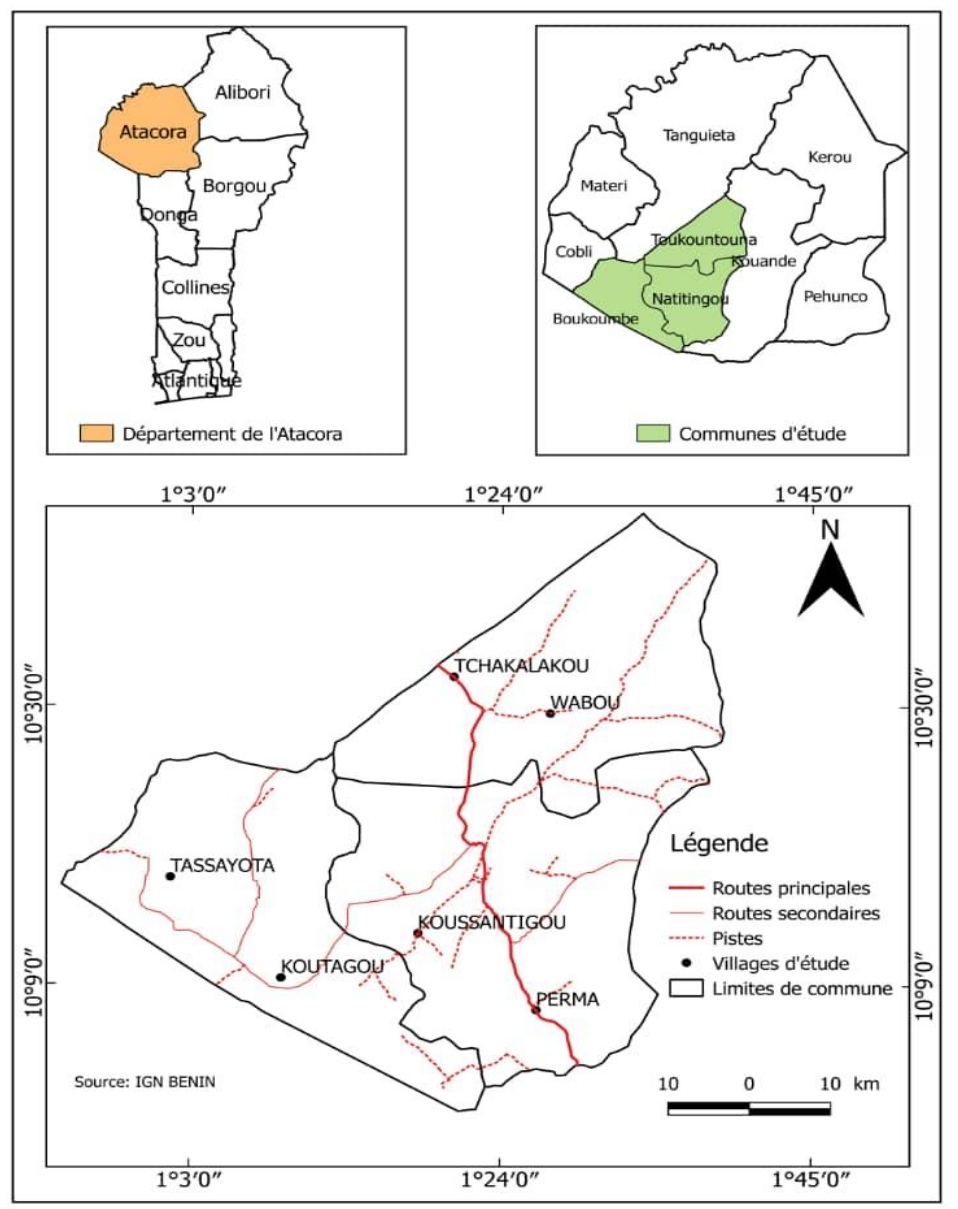

Figure 1: Carte de la zone d'étude.

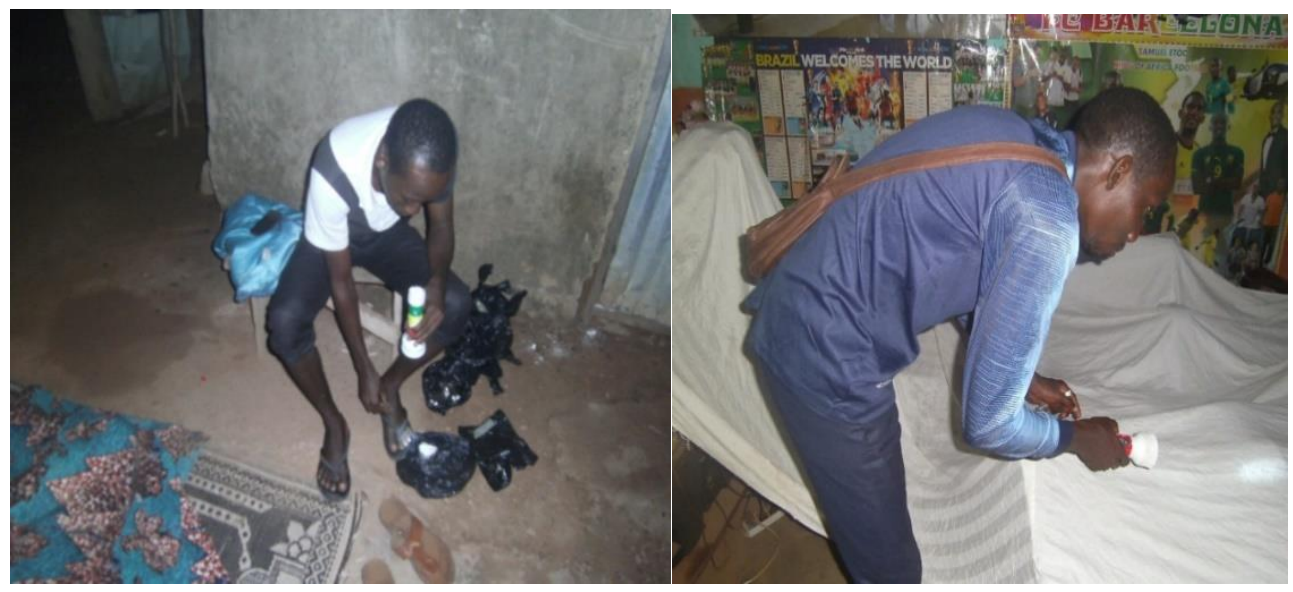

Figure 2a : Capture sur appât humain.

Figure 2b : Récolte de moustique après spray matinal. 


\section{RESULTATS}

Répartition saisonnière des espèces d'Anopheles gambiae s.l. dans la région de l'Atacora

Après les différentes captures effectuées, 1720 Anopheles gambiae s.l. ont été capturés dans l'ensemble des trois communes dont 896 anophèles en saison sèche et 824 anophèles en saison pluvieuse. Deux espèces jumelles du complexe Anopheles gambiae s.l. ont été retrouvées en sympatrie : Anopheles gambiae s.s. et Anopheles coluzzii. Le Tableau 3 montre la répartition spatiale des deux espèces en fonction des saisons avec 688 Anopheles gambiae s.s. contre 136 Anopheles coluzzii en saison pluvieuse et 516 Anopheles gambiae s.s. contre 380 Anopheles coluzzii en saison sèche (Tableau 3).

\section{Variation saisonnière des fréquences alléliques du gène Ace-1 dans le département de l'Atacora}

Le Tableau 4 présente les variations saisonnières de la fréquence allélique du gène Ace- 1 dans les différentes communes d'étude. A Natitingou, elles sont de $12,00 \%$ en saison pluvieuse contre $4,20 \%$ en saison sèche; $7,10 \%$ contre $0,90 \%$ à Toukountouna et $12,20 \%$ contre 0,60 à Boukoumbé. Globalement la f (Ace-1) est de $10,10 \%$ et $1,80 \%$ respectivement pendant la saison pluvieuse et saison sèche. Ces fréquences alléliques présentent une variation significative quelle que soit la saison ( $\mathrm{p}$-value $<0.001$ ).

\section{Variation saisonnière des fréquences alléliques du gène Kdr dans le département de l'Atacora}

Le Tableau 5 présente les variations saisonnières des fréquences alléliques du gène $K d r$ dans les localités du département de l'Atacora. f (kdr) est de 88,00\% en saison pluvieuse contre $75,90 \%$ en saison sèche à Natitingou ; $83,90 \%$ contre $80,80 \%$ à
Toukountouna et $91,30 \%$ contre $98,03 \%$ à Boukoumbé. Ainsi, pour l'ensemble des trois localités de l'Atacora la $\mathrm{f}(\mathrm{kdr})$ est de 87,30\% en saison pluvieuse contre $82,60 \%$ en saison sèche. Par ailleurs, les fréquences alléliques du gène $K d r$ présentent une variation allélique significative quelle que soit la saison ( $\mathrm{p}$-value $<0,001)$.

\section{Etude saisonnière de la panmixie}

Le Tableau 6 présente l'hétérozygotie observée $\left(H_{o}\right)$, l'hétérozygotie attendue $\left(H_{e}\right)$ et l'indice de fixation $\left(F_{I S}\right)$ chez Anopheles gambiae s.s. pour les deux loci dans les différentes localités d'étude. Pour les allèles G119S et $L 1014 F$, l'hétérozygotie observée était relativement plus faible que celle attendue pendant la saison pluvieuse dans les localités d'étude. Ce même constat est fait pendant la saison sèche pour L1014F tandis que l'hétérozygotie observée était relativement égale à celle attendue pour G119S. L'indice de fixation $F_{I S}$ varie d'une saison à une autre. Ainsi, il est plus relativement élevé en période pluvieuse qu'en période sèche pour G119S dans les différentes localités tandis qu'il est faible en période pluvieuse qu'en période sèche pour $L 1014 F$. La p-value à l'équilibre de Hardy-Weinberg permet de constater que pour G119S, toutes les sous-populations (localités) d'An. gambiae s.s. n'exprimaient un écart à la panmixie ( $\mathrm{p}$-value $>0,05$ ) quelle que soit la saison sauf celle de Natitingou en saison pluvieuse. Elle permet, par contre, de constater que pour $L 1014 F$, toutes les sous-populations (localités) d'An. gambiae s.s. exprimaient un écart à la panmixie (p-value $<0,05)$ quelle que soit la saison sauf celle de Natitingou en saison pluvieuse.

Les sous-populations d'An. gambiae s.s. présentaient un déficit d'hétérozygotie quelle que soit l'allèle en saison pluvieuse. Cependant, en saison sèche, nous avons noté un excès en hétérozygotie dans l'ensemble des 
sous-populations pour G119S tandis qu'un déficit en hétérozygotie est observé dans l'ensemble des sous-populations pour $L 1014 F$.

\section{Différenciation génétique}

Le Tableau 7 présente la différenciation génétique moyenne de la population d'Anopheles gambiae s.s. dans le département de l'Atacora. Une faible différenciation génétique est observée au sein de la population d'Anopheles gambiae s.s. quel que soit l'allèle. Les indices $F_{S T}$ liés à la mutation $L 1014 F$ du gène $K d r$ sont 0,013 et 0,006 respectivement pour les saisons pluvieuse et sèche. Ceux liés à la mutation $G 119 S$ du gène Ace- 1 sont 0.008 et 0.020 respectivement pour les saisons pluvieuse et sèche.

Tableau 3: Répartition saisonnière d'An. gambiae s.s. dans le département de l'Atacora.

\begin{tabular}{cccccc}
\hline & Localités & $\begin{array}{c}\text { saison de collecte des } \\
\text { moustiques }\end{array}$ & Effectif & $\begin{array}{c}\text { An. } \\
\text { gambiae }\end{array}$ & $\begin{array}{c}\text { An. } \\
\text { coluzzii }\end{array}$ \\
\hline & Natitingou & Saison pluvieuse & 263 & 212 & 51 \\
Département de & Toukountouna & Saison sèche & 324 & 168 & 156 \\
l'Atacora & & Saison pluvieuse & 327 & 280 & 47 \\
& & Saison sèche & 237 & 112 & 125 \\
& Boukoumbé & & & & 38 \\
& & Saison pluvieuse & 234 & 196 & 99 \\
Total & & Saison sèche & 335 & 236 & 136 \\
& & Saison pluvieuse & 824 & 688 & 380 \\
\hline
\end{tabular}

Tableau 4: Distribution des fréquences alléliques de la mutation $G 119 S$ du gène Ace-1 chez Anopheles gambiae s.s. en fonction des saisons.

\begin{tabular}{|c|c|c|c|c|c|c|c|}
\hline \multirow{2}{*}{ Localités } & \multirow{2}{*}{ Saisons } & \multirow{2}{*}{$\begin{array}{l}\text { Nombre } \\
\text { testé }\end{array}$} & \multicolumn{3}{|c|}{ Effectif } & \multirow{2}{*}{$\begin{array}{c}\text { Fréquence\% } \\
\text { F(G119S) }\end{array}$} & \multirow{2}{*}{ p-value } \\
\hline & & & $119 S / 119 S$ & $119 G / 119 S$ & $119 G / 119 G$ & & \\
\hline \multirow{2}{*}{ Natitingou } & Saison pluvieuse & 212 & 8 & 35 & 169 & 12,0 & $<0,001$ \\
\hline & Saison sèche & 168 & 0 & 14 & 154 & 4,2 & $<0,001$ \\
\hline \multirow{2}{*}{ Toukountouna } & Saison pluvieuse & 280 & 2 & 36 & 242 & 7,1 & $<0,001$ \\
\hline & Saison sèche & 112 & 0 & 2 & 110 & 0,9 & $<0,001$ \\
\hline \multirow{2}{*}{ Boukoumbé } & Saison pluvieuse & 196 & 4 & 40 & 152 & 12,2 & $<0,001$ \\
\hline & Saison sèche & 236 & 0 & 3 & 233 & 0,6 & $<0,001$ \\
\hline \multirow{2}{*}{ Total=Atacora } & Saison pluvieuse & 688 & 14 & 111 & 563 & 10,1 & $<0,001$ \\
\hline & Saison sèche & 516 & 0 & 19 & 497 & 1,8 & $<0,001$ \\
\hline
\end{tabular}


Tableau 5: Distribution des fréquences alléliques de la mutation $L 1014 F$ du gène $K d r$ chez Anopheles gambiae s.s.

\begin{tabular}{|c|c|c|c|c|c|c|c|}
\hline Localités & Saisons & $\begin{array}{l}\text { Nombre } \\
\text { testé }\end{array}$ & $1014 F / 1014 F$ & $\begin{array}{c}\text { Effectif } \\
1014 L / 1014 F\end{array}$ & $1014 L / 1014 L$ & $\begin{array}{c}\text { Fréquence \% } \\
\text { F(L1014F) }\end{array}$ & p-value \\
\hline \multirow{2}{*}{ Natitingou } & Saison pluvieuse & 212 & 167 & 39 & 6 & 88,00 & $<0,001$ \\
\hline & Saison sèche & 168 & 111 & 33 & 24 & 75,90 & $<0,001$ \\
\hline \multirow{2}{*}{ Toukountouna } & Saison pluvieuse & 280 & 203 & 64 & 13 & 83,90 & $<0,001$ \\
\hline & Saison sèche & 112 & 79 & 23 & 10 & 80,80 & $<0,001$ \\
\hline \multirow{2}{*}{ Boucoumbé } & Saison pluvieuse & 196 & 166 & 26 & 4 & 91,30 & $<0,001$ \\
\hline & Saison sèche & 236 & 189 & 39 & 8 & 88,30 & $<0,001$ \\
\hline \multirow{2}{*}{ Total=Atacora } & Saison pluvieuse & 688 & 536 & 129 & 23 & 87,30 & $<0,001$ \\
\hline & Saison sèche & 516 & 379 & 95 & 42 & 82,60 & $<0,001$ \\
\hline
\end{tabular}

Tableau 6: Variation saisonnière des hétérozygoties observées et attendues, de l'indice de fixation et de l'équilibre de Hardy-Weinberg.

\begin{tabular}{|c|c|c|c|c|c|c|c|c|}
\hline & \multicolumn{4}{|c|}{ G119S } & \multicolumn{4}{|c|}{$L 1014 F$} \\
\hline & $\boldsymbol{H}_{o}$ & $\boldsymbol{H}_{e}$ & $\begin{array}{c}F_{I S} \\
(W \& C)\end{array}$ & p-value $H W E$ & $\boldsymbol{H}_{o}$ & $\boldsymbol{H}_{e}$ & $F_{I S}(W \& C)$ & p-value $H W E$ \\
\hline & SP(SS) & SP(SS) & SP(SS) & SP(SS) & SP(SS) & SP(SS) & SP(SS) & SP(SS) \\
\hline Natitingou & $0.1651(0,0833)$ & $0.2121(0,0801)$ & $\begin{array}{c}0,2221 \\
(-0,4050)\end{array}$ & $0,0043(1,000)$ & $0.1840(0,0000)$ & $0.2121(0,2456)$ & $0,1331(1,000)$ & $0,0960(0,000)$ \\
\hline Toukountouna & $0.1286(0,0179)$ & $0.1329(0,0178)$ & $\begin{array}{c}0,0326 \\
(-0,0045)\end{array}$ & $0,6369(1,000)$ & $0.2143(0,2054)$ & $0.2799(0,3116)$ & $0,2346(0,3420)$ & $0,0004(0,0009)$ \\
\hline Boukoumbé & $0.2041(0,0127)$ & $0.2155(0,0126)$ & $\begin{array}{c}0,0529 \\
(-0,0043)\end{array}$ & $0,5020(1,000)$ & $0.1326(0,1652)$ & $0.1588(0,2063)$ & $0,1652(0,1994)$ & $0,0430(0,0060)$ \\
\hline
\end{tabular}

$F_{I S}$ : indice de fixation ; $W \& C:$ Weir \& Cockerham ; $H_{o}:$ hétérozygotie observée ; $H_{e}$ : hétérozygotie attendue ; HWE : Equilibre de Hardy weinberg ; SP : saison pluvieuse ; SS : saison sèche.

Tableau 7: Différenciation génétiques des différentes populations Anopheles gambiae s.s.

\begin{tabular}{lcc}
\hline & $F_{S T}(W \& C) L 1014 F$ & $F_{S T}(W \& C) G 119 S$ \\
$S P(S S)$ & $S P(S S)$ \\
\hline Atacora & $0,013(0.006)$ & $0,008(0.020)$ \\
\hline \multicolumn{2}{c}{ SP : saison pluvieuse ; SS : saison sèche. }
\end{tabular}




\section{DISCUSSION}

Les résultats de cette étude ont montré une répartition hétérogène des espèces $A n$. gambiae s.s., An. coluzzii dans les différentes localités d'études selon la saison. Ces différentes espèces se retrouvent en sympatrie dans les localités. Quelle que soit la saison, $A n$. gambiae s.s. reste l'espèce majoritaire. Toutefois An. coluzzii est plus représenté en saison sèche. Ceci serait probablement dû à la nature (permanente ou temporaire) des gîtes ayant favorisé l'émergence de ces moustiques et la période de capture. La représentativité relative d'An. coluzzii en saison sèche peut aussi s'expliquer par la réduction du nombre de gîtes temporaires au profit de ceux permanents. Cette observation confirme les travaux de Aïkpon et al. (2013) qui ont constaté l'abondance de An. coluzzii en période sèche. Selon Mbida et al. (2016) au Cameroun, An. coluzzii est associé aux gîtes permanents et ceux résultant de l'activité humaine alors que An. gambiae est plus inféodé aux gîtes temporaires et dépendants des pluies. Ces mêmes auteurs ont conclu que An. coluzzii préfère les collections d'eaux urbaines et s'adapte vite à la pollution. De même, la distribution saisonnière variée de ces deux espèces confirme les résultats antérieurs au Bénin qui rapportaient que les formes moléculaires $\mathrm{M}$ et $\mathrm{S}$ vivaient en sympatrie, avec la prédominance des formes $\mathrm{S}$ dans les zones de savane (Djogbénou et al., 2010). En outre, les allèles de résistance $119 \mathrm{~S}$ et $1014 \mathrm{~F}$ respectivement des gènes Ace- 1 et $K d r$ dans la région de l'Atacora ont été retrouvés, présentant une fréquence allélique plus élevée en saison pluvieuse qu'en saison sèche avec une différence significative ( $\mathrm{p}$-value < 0.05). Ce résultat peut s'expliquer par l'utilisation intensive d'insecticide (notamment les carbamates et pyréthrinoïdes) en saison des pluies pour la production agricole et accentué par l'implémentation de la pulvérisation intra domiciliaire (PID) dans l'Atacora depuis une dizaine d'année. L'utilisation massive d'insecticide constitue donc une pression de sélection de ces allèles de résistance chez les vecteurs du paludisme en Afrique. La preuve d'une relation entre l'utilisation des insecticides en agriculture et l'émergence de résistance chez les vecteurs du paludisme a été rapportée par plusieurs auteurs dont Yadouléton et al. (2010). En examinant l'impact de l'agriculture sur la résistance des vecteurs, Pierrat (2012) a noté au moins 15 espèces de vecteurs du paludisme pour lesquelles la résistance était directement liée aux traitements agricoles. Tout ceci confirme l'idée selon laquelle la résistance aux pyréthrinoïdes chez An. gambiae s.s en Afrique de l'Ouest trouve sa cause dans les traitements agricoles inadéquats, en particulier ceux appliqués au coton. Par ailleurs, il a été observé aussi que la fréquence allélique de $L 1014 \mathrm{~F}$ est plus élevée dans l'Atacora que celle de G119S quelle que soit la saison.

L'augmentation de la fréquence de l'allèle résistant du gène Ace-1 en saison pluvieuse pourrait être imputable au coût génétique lié à ce gène (Djogbénou et al., 2010).

Cette remarque dans l'Atacora est similaire à celles rapportées par Yadouleton et al., (2011) dans trois autres communes au nordest du Bénin (Parakou, à Kandi et à Banikoara) avec une prédominance remarquable du génotype homozygote résistant $1014 \mathrm{~F} / 1014 \mathrm{~F}$. L'augmentation de la fréquence allélique de la mutation $L 1014 F$ du gène $K d r$ chez les populations d'An. gambiae s.s. est inquiétante étant donné que ce gène associé à des mécanismes physiologiques de résistance, pourrait avoir un impact significatif sur l'efficacité des moustiquaires imprégnées d'insecticide à longue durée d'action distribuées et utilisées dans tout le pays pour la lutte contre le paludisme.

En effet, l'hétérozygotie observée est inférieure à l'hétérozygotie attendue $\left(\mathrm{H}_{\mathrm{o}}<\mathrm{H}_{\mathrm{e}}\right)$ par endroit pour les deux gènes, cela signifie qu'il y a plus d'homozygotes que prévus dans 
les populations étudiées. Cet excès d'homozygotes est assimilé à un risque de consanguinité au sein des sous-populations de l'Atacora. Par opposition, pour G119S, il n'a pas été noté une grande différence entre les valeurs des hétérozygoties observées et attendues chez les trois sous-populations pendant la saison sèche. Elles n'expriment donc pas un écart à la panmixie. C'est-à-dire que ces sous-populations sont en équilibre de Hardy-Weinberg $(\mathrm{P}>0.05)$ pendant la saison sèche. L'équilibre observé dans ces zones peut être lié aux effectifs échantillonnés ayant servi à l'analyse des données ou à une migration ponctuelle des individus favorisant les flux géniques et des croisements aléatoires entre les individus. En outre, les valeurs de $F_{I S}$ supérieures à zéro et importantes dans certaines zones, expriment aussi un déficit global en hétérozygotes dans les populations des localités échantillonnées. Il suggère une forte influence de la consanguinité au sein de ces sous-populations. Ce phénomène pourrait être aussi lié à la sélection généralisée de ce locus chez les différentes espèces (Gnanguenon et al., 2015) par l'action des insecticides (pyréthrinoïdes, carbamates et organophosphorés) sur les moustiques. Les individus sensibles seront éliminés au profit des résistants qui, par des accouplements préférentiels liés à l'effectif limité de leur population, vont transmettre presque exclusivement l'allèle de résistance à leurs descendants. Ils assurent de ce fait l'expansion de cette mutation génétique au sein des populations. Mis à part le hasard, trois principaux facteurs pourraient expliquer ce déséquilibre observé. Il s'agit de facteurs génétiques, l'existence d'allèles nuls et l'effet Wahlund. En ce qui concerne les causes génétiques, il est bien connu que la consanguinité (accouplement entre un individu et ses ascendants, ses collatéraux et/ou ses descendants) modifie les fréquences génotypiques. La conséquence en est une perte de variabilité génétique au cours des générations. Le second facteur pourrait être inhérent à l'existence d'allèles nuls allèles ne donnant lieu par PCR à aucune amplification. Une délétion au niveau des amorces ou une mutation dans les séquences flanquantes du gène pourraient entra,ner la présence d'allèles nuls. Enfin, le dernier facteur fait référence à la présence de sous-populations à l'intérieur de chaque population (zones agro -écologiques) pouvant induire l'effet Wahlund.

D'autre part, les valeurs des indices de différenciation obtenues dans cette étude expriment clairement une faible différenciation génétique au sein de la population d'An. gambiae s.s. de l'Atacora pour ces loci $L 1014 F$ et $G 119 \mathrm{~S}$. Nous pouvons donc dire de ces résultats, qu'il n'y a pas de différence entre ces sous populations vectrices (An. gambiae s.s.) des localités étudiées, lesquels résultats ne sont pas loin de ceux obtenus par Fassinou et al. (2019). Ils révèlent, par conséquent, de nombreux flux de gènes entre les sous populations d'An. gambiae s.s. des trois localités nonobstant la saison. Ceci grâce à l'absence probable de barrières écologiques entre ces sous populations (localités) dans la zone d'étude. Cependant, les flux de gènes ne sont pas égaux entre chaque zone, et certaines d'entre elles sont un peu plus isolées que d'autres. Aussi le type de marqueur qui a servi d'étude et la taille des échantillons peuvent aussi influencer la faible différenciation obtenue.

\section{Conclusion}

La présente étude a permis d'avoir une idée de la distribution saisonnière des espèces de An. gambiae s.s. et An. Coluzzii mais également de variabilité des fréquences alléliques des gènes de résistance Ace- 1 et $K d r$ au sein des populations de vecteurs étudiés. Ces travaux montrent que l'allèle résistant $L 1014 F$ du gène $K d r$ a été fortement sélectionné dans toutes les zones d'étude pour 
An. gambiae s.s. Par ailleurs, un déficit d'hétérozygotie est observé avec une faible différenciation génétique au sein de la population d'Anopheles gambiae s.s. de l'Atacora par rapport aux allèles de résistances quelle que soit la saison. Il est souhaitable de poursuivre les études sur la diversité génétique des vecteurs du paludisme en utilisant des marqueurs moléculaires plus résolus pour mieux établir la fine structure génétique de ces populations.

\section{CONFLIT D'INTERETS}

Les auteurs déclarent ne pas avoir de conflit d'intérêts.

\section{CONTRIBUTIONS DES AUTEURS}

RA, AM, AL, GA, SS, AD et CA ont rédigé le protocole de l'étude. RA est l'instigateur principal de l'étude et a rédigé l'article. RA et AL ont fait la collecte et l'analyse des données. Tous les auteurs ont approuvé le manuscrit final.

\section{REMERCIEMENTS}

Nous tenons à remercier le Centre de Recherche Entomologique de Cotonou qui a abrité les travaux de laboratoire. Nous remercions également l'Ecole Supérieure de Natitingou qui a soutenu matériellement et moralement ce travail.

\section{REFERENCES}

Ahadji-dabla KM, Ketoh GK, Nyamador WS, Apétogbo GY, Glitho IA. 2014. Susceptibility to DDT and pyrethroids, and detection of knockdown resistance mutation in Anopheles gambiae sensu lato in Southern Togo. Int. J. Biol. Chem. Sci., 8(1): 314-323. DOI : https://dx.doi.org/10.4314/ijbcs.v8i1.27

Ahadji-dabla KM, Ngoagouni C, Dery BD, Apétogbo GY, Ketoh GK, Glitho IA. 2019. Spatio-seasonal distribution of Anopheles gambiae sensu lato and dynamics of the Voltage gate sodium channel knock down resistance mutation (Vgsc-1014F) in the city of Lomé, Togo. Int. J. Biol. Chem. Sci., 13(3): 1654-1668. DOI :

https://dx.doi.org/10.4314/ijbcs.v13i3.36 Aïkpon R, Ago F, Ossè R, Oussou O, Aïzoun N, Oké-Agbo F, Akogbéto M. 2013. Bendiocarb resistance in Anopheles gambiae s.l. populations from Atacora department in Benin, West Africa: a threat for malaria vector control. Parasites \& Vectors, 6: $192 . \quad$ DOI: https://doi.org/10.1186/1756-3305-6-192

Aïkpon R, Sèzonlin M, Tokponon F, Okè M, Oussou O, Oké-Agbo F, Beach R, Akogbéto M. 2014. Good Performances but Short Lasting Efficacy of Actellic 50 EC Indoor Residual Spraying (IRS) on Malaria Transmission in Benin, West Africa. Parasites \& Vectors, 7: 256. DOI: https://doi.org/10.1186/1756-3305-7-256

Bimenya GS, Mugisha PS, Okwi AL, Habarulema M, Lugemwa M. 2010. Does resistance of Anopheles mosquitoes to knock-out effect of DDT deter the ability of the chemical to control malaria in Uganda. Int. J. Biol. Chem. Sci., 4(3): 657-668.

DOI: https://dx.doi.org/10.4314/ijbcs.v4i3.604 81

Corbel V, N'Guessan R, Brengues C, Chandre F, Djogbenou L, Martin T, Akogbéto M, Hougard JM, Rowland M. 2007. «Multiple Insecticide Resistance Mechanisms in Anopheles Gambiae and Culex Quinquefasciatus from Benin, West Africa ». J. Acta Tropica., 101(3): 207-216.

DOI: https://doi.org/10.1016/j.actatropica.200 7.01 .005

Darriet F. 2017. Bibliographie : Moustiquaires Imprégnées et Résistance des Moustiques aux Insecticides. IRD Éditions : Marseille.

DOI: 
http://books.openedition.org/irdeditions/ 9965

Diabate A, Baldet T, Chandre F, Akogbeto M, Guiguemde TR, Darriet F, Brengues C. 2002. The Role of Agricultural Use of Insecticides in Resistance to Pyrethroids in Anopheles Gambiae s.1. in Burkina Faso. The American Journal of Tropical Medicine and Hygiene, 67(6): 617-22. DOI:

https://doi.org/10.4269/ajtmh.2002.67.61 7

Djègbè I, Missihoun AA, Djouaka R, Akogbeto M. 2017 Surveillance Entomologique: Dynamique de la population et de la résistance aux insecticides chez Anopheles gambiae s.l; en milieu de riziculture irriguée au Sud Bénin. Journal of Applied Biosciences, 111: 10934-10943

Djogbénou L, Pasteur N, Bio-Bangana S, Baldet T, Irish SR, Akogbeto M, Weill M, Chandre.F.2010. Malaria Vectors in the Republic of Benin: Distribution of Species and Molecular Forms of the Anopheles Gambiae Complex. J. Acta Tropica, 114(2): 116-122. DOI: https://doi.org/10.1016/j.actatropica.201 0.02 .001

Fassinou AJ, Koukpo CZ, Ossè R, Agossa F, Assogba B, Sidick A, Sèwadé W, Akogbéto M, Sèzonlin M. 2019. Genetic structure of Anopheles gambiae s.s populations following the use of insecticides on several consecutive years in southern Benin. Tropical Medicine and Health, 47(1): $23 . \quad$ DOI: https://doi.org/10.1186/s41182-0190151-z.

Gillies, Coetzee M. 1987. A supplement to the Anophelinae of Africa South of the Sahara. Publ. S. Afr. Inst. Med. Res., 55: 1-143.

Gnanguenon V, Agossa FR, Badirou K, Govoetchan R, Anagonou R, Oke-Agbo
F, Azondekon R. 2015. Malaria Vectors Resistance to Insecticides in Benin: Current Trends and Mechanisms Involved. Parasites \& Vectors, 8: 223. DOI: $\quad$ https://doi.org/10.1186/s13071015-0833-2

Hemingway J, Hawkes NJ, McCarroll L, Ranson H. 2004. The Molecular Basis of Insecticide Resistance in Mosquitoes. Insect Biochemistry and Molecular Biology, 34(7): 653-665. DOI: https://doi.org/10.1016/j.ibmb.2004.03.0 18

Kelly-Hope L, Ranson H, Hemingway j. 2008. Lessons from the Past: Managing Insecticide Resistance in Malaria Control and Eradication Programmes. The Lancet Infectious Diseases, 8(6): 387-389. DOI: https://doi.org/10.1016/S14733099(08)70045-8

Martinez-Torres D, Chandre F, Williamson MS, Darriet F, Berge JB, Devonshire AL, Guillet P, Pasteur N, Pauron D. 1998. Molecular Characterization of Pyrethroid Knockdown Resistance $(K d r)$ in the Major Malaria Vector Anopheles Gambiae s.s. Insect Molecular Biology, 7(2): 179-84.

Mbida-Mbida J, Etang J, Ntonga P, Moukoko E, Awono-Ambene A, Abdou T. 2017. Nouvel aperçu sur l'écologie larvaire d'Anopheles coluzzii dans l'estuaire du Wouri, Littoral-Cameroun. Bulletin de la Société de pathologie exotique, 110: 92101.

DOI:

https://doi.org/10.1007/s13149-0160519-9

Pierrat C. 2012. Risque palustre : appréhender la vulnérabilité des individus à l'échelle locale (Sud du Bénin). VertigO - La Revue Electronique en Sciences de l'Environnement, 11: 3. DOI: http://journals.openedition.org/vertigo/11 549 
Plowe CV. 2009. The Evolution of DrugResistant Malaria. Transactions of the Royal Society of Tropical Medicine and Hygiene, 103(1): S11-S14. DOI: https://doi.org/10.1016/j.trstmh.2008.11. 002

Ranson H, N'guessan R, Lines J, Moiroux N, Nkuni Z, Corbel V. 2011. Pyrethroid Resistance in African Anopheline Mosquitoes: What Are the Implications for Malaria Control? Trends in Parasitology, 27(2): 91-98. DOI: https://doi.org/10.1016/j.pt.2010.08.004

Santolamazza F, Mancini E, Simard F, Qi Y, $\mathrm{Tu} \mathrm{Z}$, Torre A. 2008. Insertion Polymorphisms of SINE200 Retrotransposons within Speciation Islands of Anopheles Gambiae Molecular Forms. Malaria Journal, 7: 163. DOI: https://doi.org/10.1186/1475-2875-7-163

Taye A, Hadis M, Adugna N, Tilahun D, Wirtz RA. 2006. «Biting behavior and Plasmodium infection rates of Anopheles arabiensis from Sille, Ethiopia ». J. Acta Tropica, 97(1): 50-54. DOI: https://doi.org/10.1016/j.actatropica.200 5.08 .002

Weill M, Malcolm C, Chandre F, Mogensen K, Berthomieu A, Marquine M, Raymond
M. 2004. The unique mutation in Ace-1 giving high insecticide resistance is easily detectable in mosquito vectors. Insect Molecular Biology, 13: 1-7. DOI: https://doi.org/10.1111/j.13652583.2004.00452. $\mathrm{x}$

Yadouleton A, Martin T, Padonou G, Chandre F, Asidi A, Djogbénou L, Dabiré R. 2011. Cotton pest management practices and the selection of pyrethroid resistance in Anopheles gambiae population in Northern Benin. Parasites \& Vectors, 4 : 60.

Yadouleton A, N'Guessan R, Allagbé H, Asidi A, Boko M, Osse R, Padonou G, Kindé G, Akogbéto M. 2010. The impact of the expansion of urban vegetable farming on malaria transmission in major cities of Benin. Parasites \& Vectors, 3(1): 118. DOI: https://doi.org/10.1186/1756-33053-118

Yadouleton A, Klotoe JR, Agbanrin R, Ahissou F, Houndeton G, Tossou R, Agolinou A, Akogbeto M. 2018. Contrôle de qualité des rideaux imprégnés à la bifenthrine en vue de leur utilisation et vulgarisation au Bénin. Int. J. Biol. Chem. Sci., 12(5): 2044-2052. DOI: https://dx.doi.org/10.4314/ijbcs.v12i5.7 